\title{
Management and new strategy of ovarian clear cell carcinoma
}

\author{
Aikou Okamoto ${ }^{1}$ \\ Received: 20 March 2019 / Published online: 13 February 2020 \\ (c) Japan Society of Clinical Oncology 2020
}

Ovarian clear cell carcinoma (OCCC) shows unique clinical features including (1) high incidence among Asian women, particularly Japanese women, (2) low stage at presentation, (3) association with endometriosis, (4) high incidence of thromboembolic events, and (5) inherently chemo-resistant.

OCCC is likely to be diagnosed earlier than high-grade serous carcinoma (HGSC). More than half of the women with OCCC are in stage I, the 5-year survival rate of which is comparatively better at approximately $90 \%$. Nevertheless, advanced-stage OCCC is related to a highly poor prognosis, and platinum-based standard care typically accounts for a low initial response rate. However, genomic- and immunologic profiling-based strategies to target OCCC are yet to be developed.

Standard surgical treatment of patients with OCCC is the same as other EOC. Complete surgical staging is warranted to, at least, detect high-risk relapse patients. Based on the therapeutic aspect, the role of lymph node dissection remains unclear. Thus, cytoreductive surgery should be performed for OCCC patients with advanced stage or metastatic disease. For patients with stage IA OCCC who desire to preserve fertility, fertility-sparing surgery with unilateral salpingo-oophorectomy preserving contralateral normal ovary and uterus could be an option. Presently, the Japan Clinical Oncology Group is conducting the nonrandomized confirmatory study to establish the efficacy of fertility-sparing surgery for patients with stage IA OCCC or another type of epithelial ovarian carcinoma stage IC (unilateral) grade 1/2. (JCOG-1203).

Compared with HGSC, OCCC is usually resistant to conventional platinum-based chemotherapy. The Japanese Gynecologic Oncology Group conducted the first randomized phase III, OCCC-specific clinical trial (JGOG3017) that compared irinotecan and cisplatin with paclitaxel plus carboplatin in patients with OCCC. Reportedly, chemotherapy regimens in combination with irinotecan and cisplatin exhibited no advantage to a standard regimen of paclitaxel and carboplatin. Key pathways for CCC are PI3K/AKT/mTOR, angiogenesis, IL-6/STAT3, immune checkpoint, BAF, c-MET, and so on. There are closed and ongoing trials for CCC including GOG 268, GOG 254, GOG 283, ENGOT-GYN1, NRG-GY001, etc. International collaboration in clinical trials is essential to overcome the rarity of CCC. Conducting these trials and generating the evidence warrant developing novel treatments based on its molecular and immunological characteristics.

Hagiwara and Takahashi et al. recently demonstrated that ARID1A-deficient cancer cells are specifically vulnerable to inhibition of the antioxidant glutathione (GSH) and the glutamate-cysteine ligase synthetase catalytic subunit (GCLC), a rate-limiting enzyme for GSH synthesis. According to their report, ARID1A-deficient cancers are susceptible to synthetic lethal targeting of GCLC.

This discovery has enough potential to overcome CCC.

\section{Compliance with ethical standards}

Conflict of interest No author has any conflict of interest.

Publisher's Note Springer Nature remains neutral with regard to jurisdictional claims in published maps and institutional affiliations.
Aikou Okamoto

aikou@jikei.ac.jp

1 Department of Obstetrics and Gynecology, The Jikei University School of Medicine, 3-25-8 Nishi-Shimbashi, Minato-ku, Tokyo 105-8461, Japan 